Pacific Journal of Mathematics

OBSTRUCTIONS TO PSEUDOISOTOPY IMPLYING ISOTOPY 


\section{OBSTRUCTIONS TO PSEUDOISOTOPY IMPLYING ISOTOPY FOR EMBEDDINGS}

\section{Kenneth C. Millett}

A key theorem in efforts to determine the homotopy groups of spaces of automorphisms and embeddings has been Morlet's "disjonction theorem" or, equivalently, the author's "concordance implies isotopy theorem." Although it was known that the conclusions of these theorems could not be true without some restrictive hypotheses the only explicit nonzero obstructions occurred in the, possibly anomalous, cases of codimensions $0,1,2$ and $n$. In this paper nonzero obstructions are determined for each of the remaining cases showing that the theorems are best possible.

Let $I=[-1,1], D^{p}=I^{p}, S^{p}=\partial D^{p+1}$, and, for any compact piecewise linear manifold $M$, let the symbol $\sqcup$ denote the subset $M \times\{-1\} \cup$ $\partial M \times I$ of $M \times I$. Given piecewise linear manifolds $(M, \partial M) \subset(N, \partial N)$ and a subset $A$ of $M$, let $E(M, N ; \operatorname{rel} A)$ denote the $\Delta$-set of locally flat piecewise linear embeddings of $(M, \partial M$, int $M)$ in $(N, \partial N$, int $N)$ which are fixed on $A$.

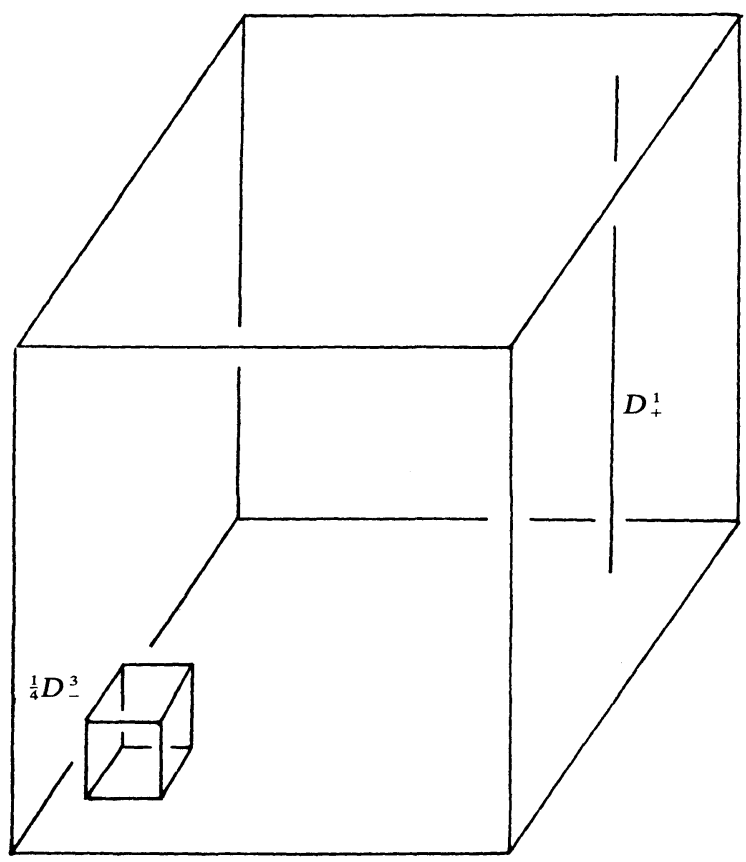

Figure 1. $\left.D_{+}^{1} \subset D^{3}\right|_{4} ^{\frac{1}{4}} D_{-}^{3}$ 
In order to describe the obstructions we require an explicit description of the manifolds in which they arise. Thus let $D_{+}^{p}=$ $\left(\frac{1}{2}, \cdots, \frac{1}{2}\right) \times D^{p} \subset D^{n}, \quad{ }_{4}^{1} D_{-}^{n}=\left\{\left(x_{1}, \cdots, x_{n}\right) \in D^{n}\left|\max _{i}\right| x_{i}+\frac{1}{2} \mid \leqq \frac{1}{4}\right\}, \quad$ and choose a piecewise linear homeomorphism $h: D^{n} \backslash$ int ${ }_{4}^{1} D_{-}^{n} \rightarrow S^{n-1} \times I$. We shall consider the embedding of $D^{p}$ as a submanifold of $S^{n-1} \times I$ given by

$$
D^{p} \rightarrow D_{+}^{p} \hookrightarrow\left(D^{n} \backslash \operatorname{int}_{4}^{1} D_{-}^{n}\right) \stackrel{h}{\rightarrow} S^{n-1} \times I .
$$

Finally, let $\sum_{i, n}^{k}: \Pi_{i}\left(S^{n}\right) \rightarrow \Pi_{i+k}\left(S^{n+k}\right)$ denote the $k$-fold suspension homomorphism. The precise statement of the result is:

THEOREM 1.1. If $n \geqq 5$ and $n-p \geqq 3$, there is an epimorphism of

$$
\prod_{2 n-p-4}\left(E\left(D^{p} \times I,\left(S^{n-1} \times I\right) \times I ; \text { rel } \sqcup\right)\right)
$$

onto the following nonzero groups; if $n \neq 8, \operatorname{ker} \sum_{2 n-3, n-1}^{n-p-2}$, if $n=8$ and $p=5, \mathbf{Z}$, and if $n=8$ and $p<5, \mathbf{Z}_{2}$.

Because the above group is never zero, the theorem implies that the following theorems are best possible.

Theorem 1.2. (Morlet's "Lemme de Disjonction", $[1,7]) . \quad$ Let $D^{p}$ and $D^{q}$ be disjoint submanifolds of $N$ with $n-p \geqq 3$, then

$$
\prod_{i}\left(E\left(D^{p} \times I, N \times I ; \text { rel } \sqcup\right), E\left(D^{p} \times I,\left(N \backslash D^{q}\right) \times I ; \text { rel } \sqcup\right)\right)=0
$$

for $i \leqq 2 n-p-q-4$

TheOREM 1.3 ("Concordance implies Isotopy", [5]). Let $D^{p}$ be a submanifold of $N, n-p \geqq 3$, and $\Pi_{j}(N)=0$ for $j \leqq k$, then

$$
\prod_{i}\left(E\left(D^{p} \times I, N \times I ; \text { rel } \sqcup\right)\right)=0
$$

for $i \leqq n+k-p-3$.

Letting $N=S^{n-1} \times I$, so that $k=n-2$, Theorem 1.1 shows immediately that Theorem 1.3 is best possible. Letting $D^{1}=$ $* \times I \subset S^{n-1} \times I=N$, the fact (proved via an Alexander isotopy argument, cf. $[1,5])$ that 


$$
\prod_{i}\left(E\left(D^{p} \times I,\left(N \backslash D^{1}\right) \times I ; \text { rel } \sqcup\right)\right)=0
$$

implies, via the exact sequence of the pair, that Theorem 1.2 is best possible.

The proof of Theorem 1.1 consists of a reduction to a homotopy theoretic problem using a result of [6]. This homotopy problem is then analyzed via classical techniques involving the homotopy groups of wedges of spheres, Whitehead products, and the suspension homomorphism.

I wish to thank L. Larmore for several helpful conversations.

\section{The Calculation.}

Let $\operatorname{Aut}(M ; \operatorname{rel} A)$ denote the $\Delta$-set of piecewise linear automorphisms of a manifold $M$ which are fixed on the subset $A$. Consider the fibrations

$$
\begin{array}{ll}
\operatorname{Aut}\left(D^{n} \times I ; \text { rel } \cup \cup\left(* \cup D_{+}^{p}\right) \times I\right) & \operatorname{Aut}\left(D^{n} \times I ; \operatorname{rel} \cup \cup\left(* \cup D_{+}^{p}\right) \times I\right) \\
\operatorname{Aut}\left(D^{n} \times I ; \operatorname{rel} \cup \cup * \times I\right) & \operatorname{Aut}\left(D^{n} \times I ; \operatorname{rel} \sqcup \cup D_{+}^{p} \times I\right) \\
\qquad\left(D^{p} \times I,\left(D^{n} \backslash *\right) \times I ; \text { rel } \sqcup\right) & E\left(I_{1}\left(D_{+}^{n} \backslash D^{p}\right) \times I ; \operatorname{rel}\{-1\}\right)
\end{array}
$$

where $*=\left(-\frac{1}{2}, \cdots,-\frac{1}{2}\right)$ and $D_{+}^{p}=\left(\frac{1}{2}, \cdots, \frac{1}{2}\right) \times D^{p} \subset D^{n}$. The base spaces

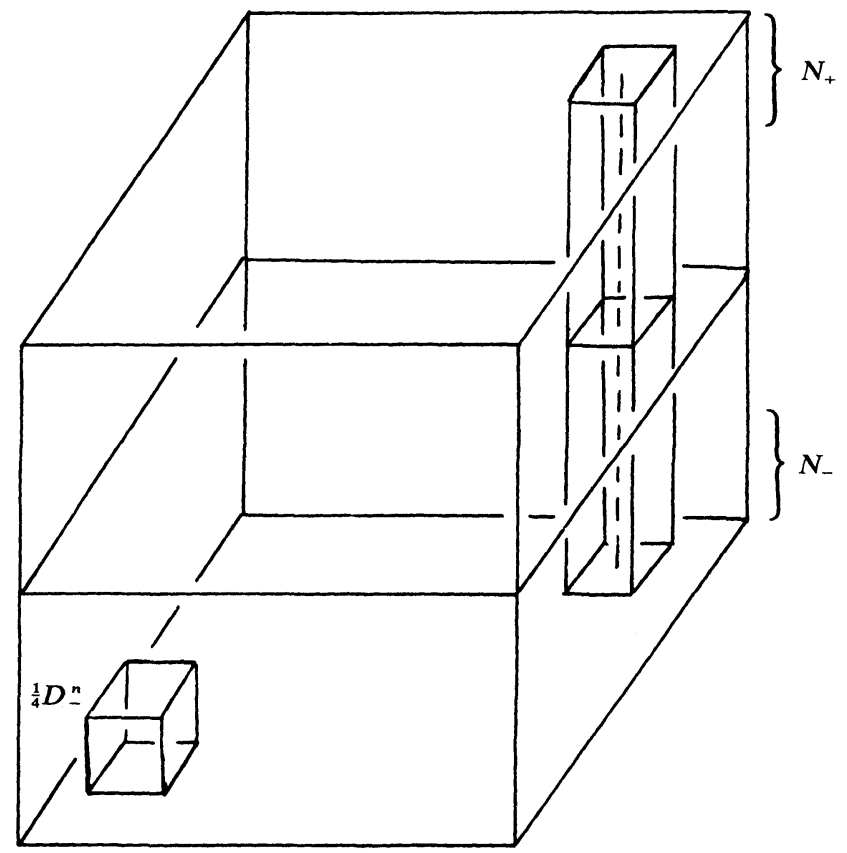

FiguRE 2. $* \in$ Model for $\left(S^{n-p-1} \times D^{p+1}\right) \times I=N$. 
are homotopy equivalent to $E\left(D^{p} \times I,\left(S^{n-1} \times I\right) \times I\right.$; rel $\left.\sqcup\right)$ and $E\left(I,\left(S^{n-p-1} \times D^{p+1}\right) \times I\right.$; rel $\left.\{-1\}\right)$, respectively, where $D^{p} \subset S^{n-1} \times I \subset$ $D^{n} \backslash *$ as described in the introduction, and $I=$ $* \times I \subset\left(S^{n-p-1} \times D^{p+1}\right) \times I \subset\left(D^{n} \backslash D^{p}\right) \times I$. Since both total spaces are contractible and the fibers are equal, the homotopy groups of these two complexes are isomorphic. This it suffices to show that, for $n \geqq 5$,

$$
\prod_{2 n-p-4}\left(E\left(I,\left(S^{n-p-1} \times D^{p+1}\right) \times I ; \operatorname{rel}\{-1\}\right)\right) \neq 0 .
$$

Let $N=S^{n-p-1} \times D^{p+1}, N_{+}=S^{n-p-1} \times D^{p} \times[0,1], N_{-}=S^{n-p-1} \times D^{p} \times$ $[-1,0], * \in$ interior ${ }_{4}^{1} D_{-}^{n} C$ interior $N_{-}$, and $\hat{N}=N \backslash\left(\right.$ interior ${ }_{4}^{1} D_{-}^{n}$ ).

It follows from the splitting of the exact homotopy sequence of the fibration,

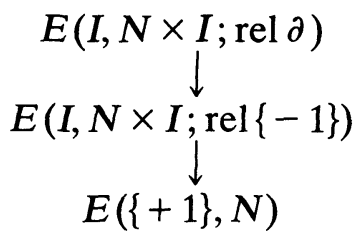

and the fact that $E(\{+1\}, N)$ has the homotopy type of $N$, that $\Pi_{i}(E(I, N \times I ;$ rel $\partial))$ is isomorphic to $\Pi_{i+1}(N) \oplus \Pi_{i}(E(I, N \times I$; $\operatorname{rel}\{-1\}))$.

By Theorem 3.18 [6] there is a homomorphism

$$
\prod_{i}(E(I, N \times I ; \text { rel } \partial)) \rightarrow \prod_{i+1}\left(h_{\partial}(N), h_{\partial}(N, *)\right)
$$

which is an isomorphism for $i \leqq 2 n-p-5$ and an epimorphism for $i=2 n-p-4$. Here $h_{\partial}(N)$ denotes the $\Delta$-set of homotopy equivalences of $N$ which are fixed on $\partial N$ and $h_{\partial}(N, *)$ denotes the sub $\Delta$-set of those homotopy equivalences which preserve the basepoint $*$ and its complement in $N$.

The homomorphism can be defined as follows: Let $f: D^{i} \times I \rightarrow D^{i} \times$ $(N \times I)$ represent an element of $\Pi_{i}(E(I, N \times I$; rel $\partial))$. By the isotopy extension theorem [4], there is an extension of $f, F_{0}: D^{i} \times(N \times I) \rightarrow D^{i} \times$ $(N \times I)$ such that $F_{0} \mid D_{-}^{i} \times(N \times I)=1$ and $F_{0} \mid D^{i} \times \partial(N \times I)=1$. A homotopy, $F_{t}$, is then constructed, respecting the $D^{\prime}$ coordinates, such that $F_{t}\left|D_{-}^{i} \times(N \times I)=1, \quad F_{t}\right| D^{i} \times \partial(N \times I)=1$, $F_{t} \mid D_{+}^{i} \times((N \backslash *, *) \times I) \rightarrow D_{+}^{i} \times((N \backslash *, *) \times I)$, and such that $F_{1}$ respects the $I$-coordinate. $F_{1}$ then represents an element of 
$\Pi_{i}\left(\Omega h_{\partial}(N), \Omega h_{\partial}(N, *)\right)$ whose image via the isomorphism to $\Pi_{i+1}\left(h_{\partial}(N), h_{\partial}(N, *)\right)$ is defined to be the image of $[f]$.

The remainder of the paper is devoted to showing that $\Pi_{i+1}\left(h_{\partial}(N), h_{\partial}(N, *)\right)$ is isomorphic to $\Pi_{i+1}(N)$ for $i \leqq 2 n-p-5$, verifying Theorems 1.2 and 1.3 in this special case, and showing that if $n \geqq 5$, $\Pi_{2 n-p-3}\left(h_{\partial}(N), h_{\partial}(N, *)\right)$ is isomorphic to $\Pi_{2 n-p-3}(N)$ plus a nontrivial group, proving Theorem 1.1.

The determination of $\Pi_{i}\left(h_{\partial}(N), h_{\partial}(N, *)\right)$ is rather indirect and depends critically upon the specific $N$ under consideration. The basic idea is to analyze the exact sequence,

$$
\rightarrow \prod_{i+1}\left(h_{\partial}(N, *), h_{\partial}(\hat{N})\right) \rightarrow \prod_{i+1}\left(h_{\partial}(N), h_{\partial}(\hat{N})\right) \rightarrow \prod_{i+1}\left(h_{\partial}(N), h_{\partial}(N, *)\right) \rightarrow
$$

What we actually do is describe an exact sequence for $\Pi_{i+1}\left(h_{\partial}(N), h_{\partial}(\hat{N})\right)$ and an analogous sequence for $\Pi_{i+1}\left(h_{\partial}(N, *), h_{\partial}(\hat{N})\right)$ which includes as direct summand. The cokernel of this inclusion of sequences gives an exact sequence for $\Pi_{i+1}\left(h_{\partial}(N), h_{\partial}(N, *)\right)$ and reduces the computation to classical homotopy theory.

The first step is to consider the fibration (onto the arc component of the identity)

$$
h_{\partial \cup n b d(*)}(N, *) \rightarrow h_{\partial}(N, *) \underset{g}{\rightarrow} g h\left(D^{n}, *\right)
$$

where $g$ is "taking the germ at $*$ ", and $g h\left(D^{n}, *\right)$ denotes the germs of homotopy equivalence of $\left(D^{n}, *\right)$ at $* . h_{\partial \cup n b d(*)}(N, *)$ is homotopy equivalent to $h_{\partial}(\hat{N})$ and $g h\left(D^{n}, *\right)$ is homotopy equivalent to $h\left(S^{n-1}\right)$. Thus $\Pi_{i+1}\left(h_{\partial}(N, *), h_{\partial}(\hat{N})\right)$ is isomorphic to $\Pi_{i+1}\left(h\left(S^{n-1}\right)\right)$ which occurs in the following exact sequence

$$
\rightarrow \prod_{i+1}\left(h\left(S^{n-1}\right)\right) \stackrel{e v}{\longrightarrow} \prod_{i+1}\left(S^{n-1}\right) \stackrel{\partial}{\rightarrow} \prod_{i+n-1}\left(S^{n-1}\right) \rightarrow
$$

Here $e v$ denotes the evaluation at a basepoint and the $\partial$ homomorphism is $\partial(\alpha)=\left[\alpha, \iota_{n-1}\right]$, the Whitehead product with the generator $\iota_{n-1}$ of $\Pi_{n-1}\left(S^{n-1}\right)$.

To describe the sequence for $\Pi_{i+1}\left(h_{\partial}(N), h_{\partial}(\hat{N})\right)$ we consider the exact sequence

$$
\rightarrow \prod_{i+1}\left(h_{\partial}(N), h_{\partial}(\hat{N})\right) \rightarrow \prod_{i}\left(h_{\partial}(\hat{N})\right) \stackrel{\lrcorner}{\leftrightarrows} \prod_{i}\left(h_{\partial}(N)\right) \rightarrow
$$


which splits via the homomorphism $j$ induced by taking $N$ to $N_{+}$and extending homotopy equivalences of $N_{+}$to $\hat{N}$ by the identity on the complement. We shall show that there are exact sequences for $h_{\partial}(\hat{N})$ and $h_{\partial}(N)$ which respect this splitting and therefore determine a sequence for $\Pi_{i+1}\left(h_{\partial}(N), h_{\partial}(\hat{N})\right)$. Recall that $N=S^{n-p-1} \times D^{p+1}$ so that we have an exact sequence similar to the previous one,

$$
\rightarrow \prod_{i}(h(N)) \stackrel{e v}{\longrightarrow} \prod_{i+p+1}\left(S^{n-p-1}\right) \stackrel{\partial}{\rightarrow} \prod_{i+n-1}\left(S^{n-p-1}\right) \rightarrow
$$

where $e v$ is evaluation on $* \times D^{p+1}$ for a base point $*$ in $S^{n-p-1}$ and $\partial$ is the Whitehead product with $\iota_{n-p-1}$.

To develope a similar sequence for $\left.\Pi_{i}\left(h_{\partial}\right)(\hat{N})\right)$ we consider the two stage cofibration decomposition

$$
\begin{aligned}
S^{i+p} \vee S^{i} \stackrel{a_{2}^{i}}{\longrightarrow} & X_{2}^{i}=S^{i} \times\left(S^{n-p-1} \times S^{p} \cup S^{n-1}\right) \cup * \times \hat{N} \\
& \downarrow_{2} \\
S^{i+n-1} \stackrel{a_{1}^{i}}{\longrightarrow} & X_{1}^{i}=X_{2}^{i} \cup S^{i} \times\left(* \times D^{p+1} \cup \tilde{D}^{1}\right) \underset{p_{2}}{\longrightarrow} S^{i+p+1} \vee S^{i+1} \\
& \downarrow_{1} \\
& X_{0}^{i}=S^{i} \times \hat{N} \underset{p 1}{\longrightarrow} S^{i+n}
\end{aligned}
$$

illustrated in Figure 3, where $i_{j}$ is the inclusion, $p_{j}$ is the quotient map, and $a_{j}^{i}$ is the attaching map of the cell(s). Also, given a map $f: A \rightarrow Y$ defined on a set $A \subset X$, let $[X, Y]_{f}$ denote the set of homotopy classes of extensions of $f$ to $X$.

One then has an exact sequence,

$$
\begin{aligned}
& {\left[X_{0}^{i}, \hat{N}\right]_{p i_{1}} \cong \prod_{i+n}(\hat{N})} \\
& {\left[X_{0}^{i}, \hat{N}\right]_{p p_{12}} \cong \prod_{i}\left(h_{\partial}(\hat{N})\right)} \\
& {\left[X_{1}^{i}, \hat{N}\right]_{p i_{12}} \cong \prod_{i+p+1}(\hat{N}) \oplus \prod_{i+1}(\hat{N})} \\
& \downarrow \\
& {\left[\begin{array}{c}
X_{0}^{i-1}, \hat{N} \\
\downarrow
\end{array}\right]_{p i_{1}} \cong \prod_{i+n-1}(\hat{N})}
\end{aligned}
$$




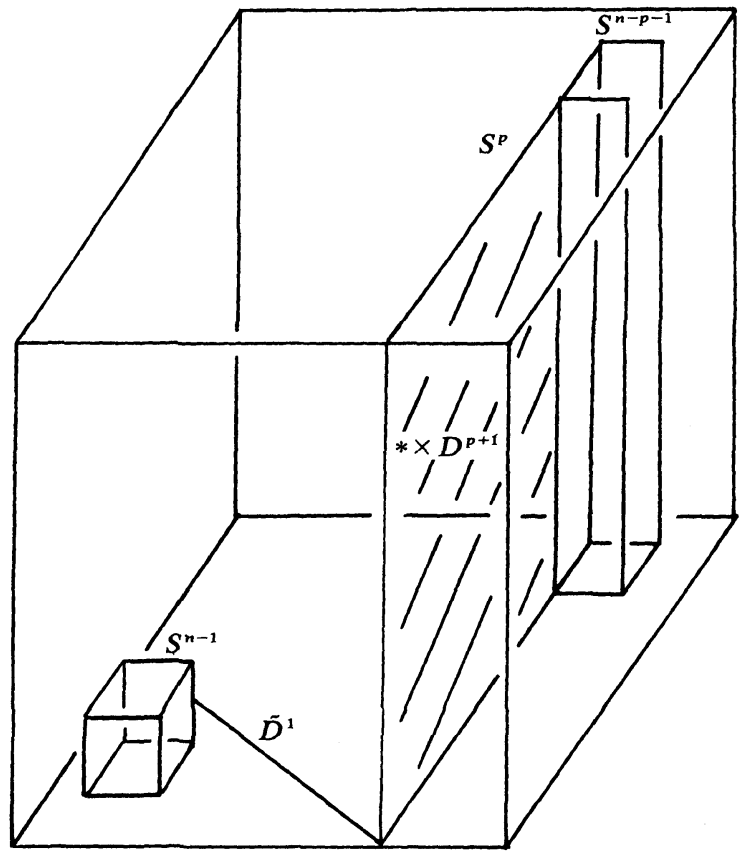

Figure 3. Model for $\tilde{D}^{1}, * \times D^{p+1} \subset \hat{N}$.

where the two unlabeled functions are induced by inclusions and $p$ is the projection to $\hat{N}$. From the first cofiber sequence we observe that $\left[X_{0}^{i-1}, \hat{N}\right]_{p i_{1}}$ is isomorphic to $\Pi_{i+n-1}(\hat{N})$, from the second we observe that $\left[X_{1}^{i}, \hat{N}\right]_{p i i_{2}}$ is isomorphic to $\Pi_{i+p+1}(\hat{N}) \bigoplus \Pi_{i+1}(\hat{N})$, while $\left[X_{0}^{i}, \hat{N}\right]_{p i i_{2}}$ is, by construction, $\Pi_{i}\left(h_{\partial}(\hat{N})\right)$. The "boundary" homomorphism takes $[f] \in$ $\left[X_{1}^{i}, \hat{N}\right]_{p i_{12} i_{2}}$ to $\left[f \circ \alpha_{1}^{i}\right] \in \Pi_{i+n-1}(\hat{N})=\left[X_{0}^{i-1}, \hat{N}\right]_{p i_{1} \text {. }}$

To describe the associated sequence for $\Pi_{i}\left(h_{\partial}(N), h_{\partial}(\hat{N})\right)$ we require precise information relating the sequences and the splitting. First, $\hat{N}$, has the homotopy type of $S^{n-p-1} \vee S^{n-1}$ so that we must consider the sequence

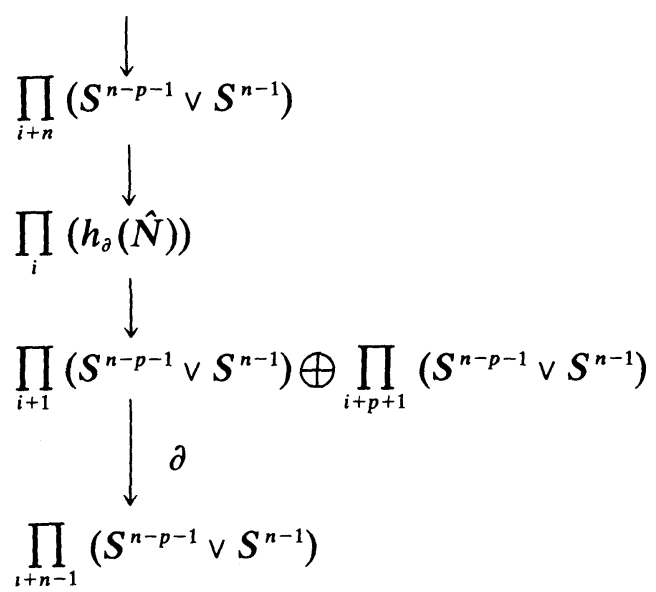


and provide an explicit description of $\partial$. To achieve this we employ the theorem of Hilton [3] describing the homotopy groups of wedges of spheres. Let $\iota_{n-p-1}$ and $\iota_{n-1}$ denote the homotopy classes of the inclusions of $S^{n-p-1}$ and $S^{n-1}$ into $S^{n-p-1} \vee S^{n-1}$, respectively. Following Hilton one defines basic Whitehead products of weight 1 as $\iota_{n-p-1}$ and $\iota_{n-1}$ with the ordering $\iota_{n-p-1}<\iota_{n-1}$. Given basic products of weight less than $w$, a basic product of weight $w$, greater than 1 , is a product $[a, b]$ where $a$ and $b$ are basic products whose weights add to $w, a<b$, and if $b$ is a Whitehead product $[c, d]$, then $c \leqq a$. Basic products are ordered by weight and, except for $w=1$, arbitrarily within a given weight. A Whitehead product $\rho$ involving $k \iota_{n-p-1}$ 's and $l \iota_{n-1}$ 's is a specific map from $S^{h(\rho)+1}$ into $S^{n-p-1} \vee S^{n-1}$ where $h(\rho)=k(n-p-2)+l(n-2)$. Hilton's theorem states that, if $n-1$ and $n-p-1$ are at least 2,

$$
\prod_{i}\left(S^{n-p-1} \vee S^{n-1}\right) \cong \underset{\text { basic products } \rho}{\bigoplus_{i}} \prod\left(S^{h(\rho)+1}\right)
$$

where the isomorphism is given by taking $\alpha \in \Pi_{i}\left(S^{h(\rho)+1}\right)$ to $\rho \circ \alpha$.

The boundary homomorphism takes an element $\beta \in$ $\Pi_{i+1}\left(S^{n-p-1} \vee S^{n-1}\right)$ to $\left[\iota_{n-1}, \beta\right] \in \Pi_{i+n-1}\left(S^{n-p-1} \vee S^{n-1}\right)$ and an element $\gamma \in$ $\Pi_{i+p+1}\left(S^{n-p-1} \vee S^{n-1}\right)$ to $\left[\iota_{n-p-1}, \gamma\right] \in \Pi_{i+n-1}\left(S^{n-p-1} \vee S^{n-1}\right)$. We shall only describe the argument for $\beta$ as the case for $\gamma$ is quite similar. Given $\beta \in \Pi_{i+1}\left(S^{n-p-1} \vee S^{n-1}\right)$, represented by a map $g:\left(D^{i} \times D^{1}\right.$, $\left.\partial\left(D^{i} \times D^{1}\right)\right) \rightarrow\left(S^{n-p-1} \vee S^{n-1}, *\right)$, one defines an associated map $\tilde{g}:\left(S^{i} \times\right.$ $\left.D^{1}, S^{i} \times S^{0}\right) \rightarrow\left(S^{n-p-1} \vee S^{n-1}, *\right)$ since $S^{i-1} \times D^{1}$ goes to $*$. The element $[f] \in\left[X_{1}^{i}, N\right]_{i i_{2}}$ which corresponds to $\beta$ is defined by taking $\tilde{g}$ on $S^{i} \times D^{1}$, the constant map on $S^{i} \times D^{p+1}$, and projection to $\hat{N}$ on the remainder of $X_{1}^{i}$. To show that the composition $\left[f \circ a_{1}^{i}\right]$ is $\left[\iota_{n-1}, \beta\right]$ we must use the geometry of $X_{1}^{i}$ to provide a description of $\left[f \circ a_{1}^{i}\right]$ which makes this clear. The map $a_{1}^{i}$ on $S^{i+n-1}=\partial\left(D^{i} \times D^{n}\right)=S^{i-1} \times D^{n} \cup D^{i} \times S^{n-1}$ is given by taking $S^{i-1} \times D^{n}$ to $* \times \hat{N}$ in $X_{1}^{i}$ by the projection to the top cell of $\hat{N}$ and by taking $D^{i} \times S^{n-1}$ into $X_{1}^{i}$ by projecting $D^{i}$ to $S^{i}$ on the first factor and taking the attaching map of the top cell of $\hat{N}$ on the second factor. When composed with $f$ this gives projection onto $S^{n-1}$ in $S^{n-p-1} \vee S^{n-1}$ on $S^{i-1} \times D^{n}=\left(S^{i-1} \times D^{1}\right) \times D^{n-1}$ where $D^{1}$ corresponds to the $D^{1}$ in the decomposition of $\hat{N}$. On $D^{i} \times S^{n-1}=\left(D^{i} \times S^{0}\right) \times D^{n-1} \cup$ $D^{i+1} \times S^{n-2}$ the composition is given by projection to the $S^{n-1}$ on the first part of the union, as above, and by $\beta$ on the second part of the union. Noting that $\left(S^{i-1} \times D^{1}\right) \times D^{n-1} \cup\left(D^{i} \times S^{0}\right) \times D^{n-1}=S^{i} \times D^{n-1}$ we see that the composition is given by projection to the $S^{n-1}$ on $S^{i} \times D^{n-1}$ and by $\beta$ on $D^{i+1} \times S^{n-2}$, which is precisely the definition of $\left[\iota_{n-1}, \beta\right]$.

We are now able to describe $\Pi_{i+1}\left(h_{\partial}(N), h_{\partial}(\hat{N})\right)$ by considering the 
commutative diagram of exact sequences

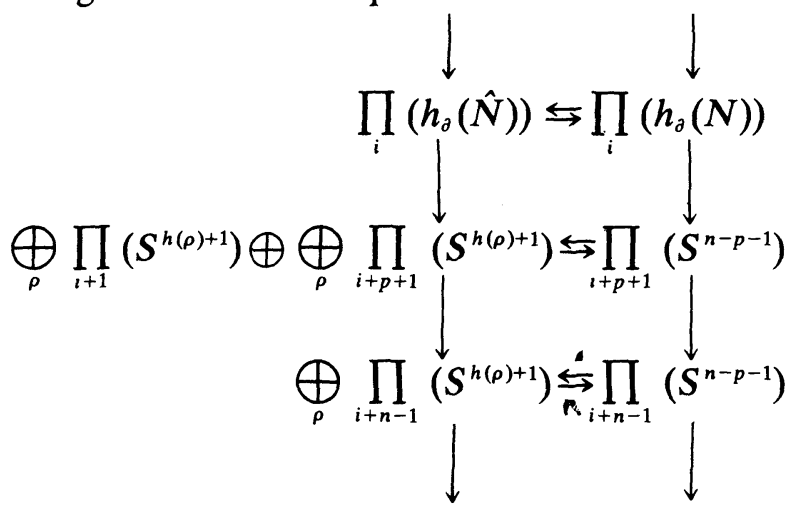

where the inclusion of the right hand sequence is given by the splitting homomorphism at the top and as the $\rho=\iota_{n-p-1}$ summand in the other cases. By the calculations above, this commutes with the boundary homomorphisms and is a splitting of the left sequence. As a consequence we have the following exact sequence

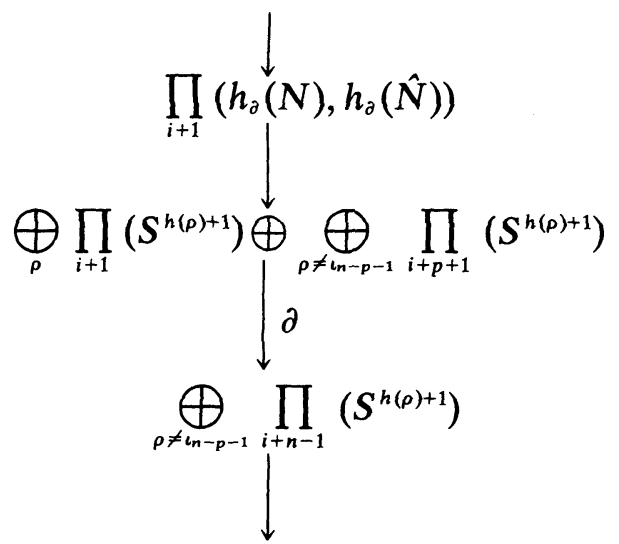

with $\partial$ computed above. Finally, returning to the exact sequence of the triple $\left(h_{\partial}(N), h_{\partial}(N, *), h_{\partial}(\hat{N})\right)$ we have a commutative diagram of exact sequences,

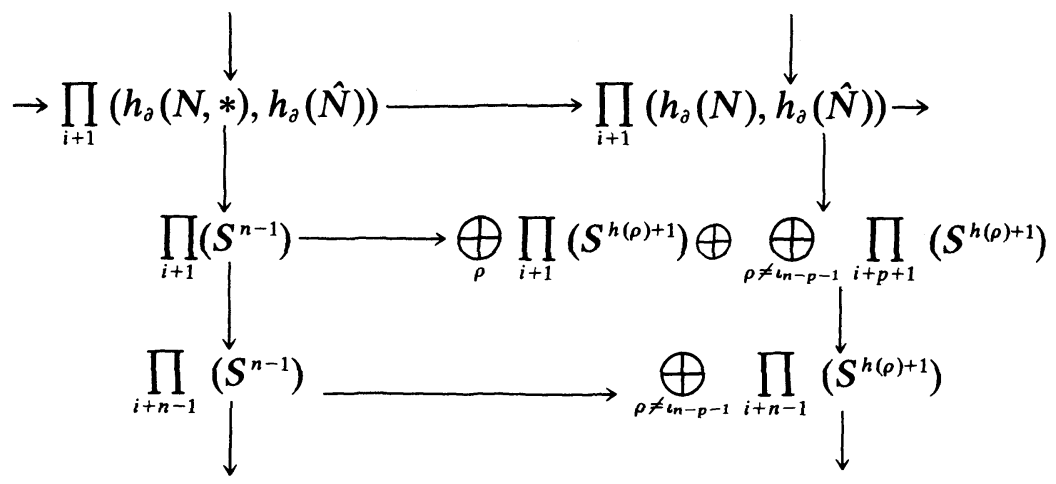


where the lower two homomorphisms are the injection as the summand. Thus we have the following exact sequence for $\Pi_{i+1}\left(h_{\partial}(N), h_{\partial}(N, *)\right)$ which we wish to analyze when $i \leqq 2 n-p-4$,

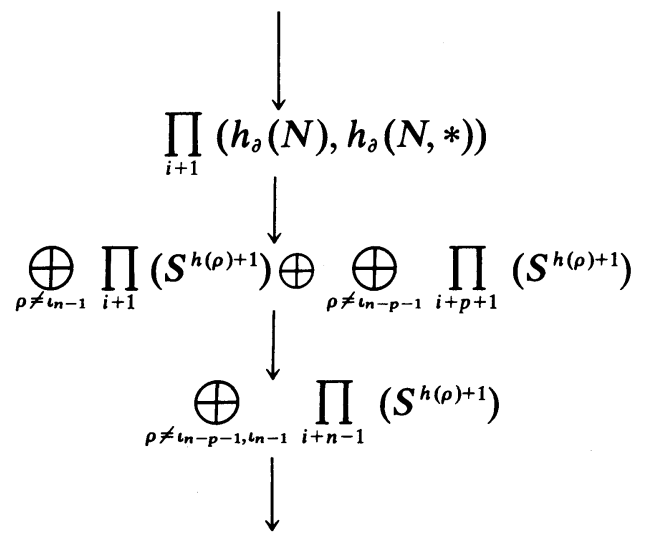

The next lemma provides for a substantial simplification of the exact sequence in this range.

LEMMA. If $\alpha$ or $\beta$ are suspension elements $\Sigma \alpha^{\prime}$ or $\Sigma \beta^{\prime}$, respectively, then

(i) $\left[\iota_{n-1}, \rho \circ \alpha\right]=\left[\iota_{n-1}, \rho\right] \circ \Sigma^{n-1} \alpha^{\prime}$, and

(ii) $\left[\iota_{n-p-1}, \rho \circ \beta\right]=\left[\iota_{n-p-1}, \rho\right] \circ \Sigma^{n-p-2} \beta^{\prime}$, respectively.

Proof. This follows immediately from the commutativity of the following diagram for $\alpha^{\prime}$,

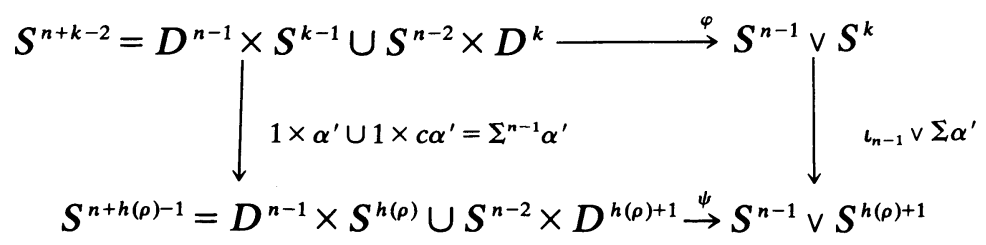

where $c \alpha^{\prime}$ denotes the cone on $\alpha^{\prime}$ and the horizontal maps, $\varphi$ and $\psi$, are those used to define the Whitehead products. The argument for $\beta$ is just the same.

We shall also need the suspension theorem, cf. [2], which we paraphrase as follows:

Suspension Theorem. The suspension homomorphism,

$$
\Sigma: \prod_{i}\left(S^{k}\right) \rightarrow \prod_{i+1}\left(S^{k+1}\right)
$$


is an isomorphism for $i<2 k-1$ and an epimorphism for $i=2 k-1$ with kernel generated by $\left[\iota_{k}, \iota_{k}\right]$. This kernel is infinite cyclic if $k$ is even, is $\mathbf{Z}_{2}$ if $k$ is odd unequal to 1,3 , or 7 , and is zero if $k$ is 1,3 , or 7 .

Recall that $h(\rho)=k(n-p-2)+l(n-2)$ when $\rho$ involves $k \iota_{n-p-1}$ 's and $l \iota_{n-1}$ 's. Thus an element of $\Pi_{j+1}\left(S^{h(\rho)+1}\right)$ is a suspension if $j \leqq$ $2 h(\rho)-1$. For $j=i$ or $i+p$ this can fail only for $\rho=\iota_{n-p-1}$ or $\iota_{n-1}$. By the lemma the other elements are taken to the appropriate basic product summands by an iterated suspension which, by the suspension theorem, gives an isomorphism. Thus, for $i \leqq 2 n-p-4$, we have the following exact sequence

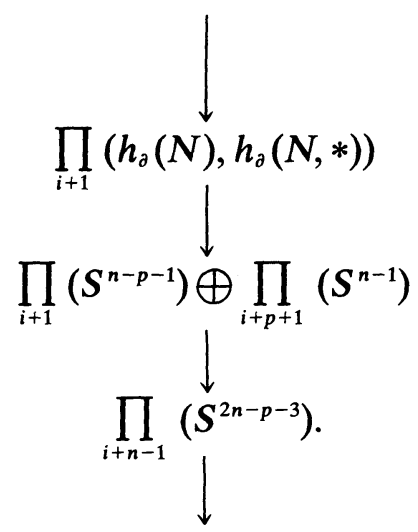

The isotopy extension theorem [4] for a point $* \subset N$ shows that $\Pi_{i+1}\left(h_{\partial}(N), h_{\partial}(N, *)\right)$ is isomorphic to $\Pi_{i+1}(N) \oplus \Pi_{i+1}\left(\left(h_{\partial} N\right)_{*}, h_{\partial}(N, *)\right)$ where $h_{\partial}(N)_{*}$ is the fiber of the evaluation $h_{\partial}(N) \rightarrow N$. Moreover $\Pi_{i+1}(N)$ is isomorphic to $\Pi_{i+1}\left(S^{n-p-1}\right)$ and goes isomorphically onto the summand in the sequence. The first case where the elements of $\Pi_{i+p+1}\left(S^{n-1}\right)$ could fail to be suspensions occurs when $i+p+1=2 n-3$, i.e., $i=2 n-p-4$. By the suspension theorem $\partial$ takes $\Pi_{i+p+1}\left(S^{n-1}\right)$ isomorphically onto $\Pi_{i+n-1}\left(S^{2 n-p-3}\right)$ for $i<2 n-p-4$.

In the case $i=2 n-p-4$ the suspension theorem implies that the suspension elements of $\Pi_{2 n-3}\left(S^{n-1}\right)$ go onto $\Pi_{3 n-p-5}\left(S^{2 n-p-3}\right)$ so that $\Pi_{i+1}\left(h_{\partial}(N)_{*}, h_{\partial}(N, *)\right)=0$ for $i<2 n-p-4$. To complete the proof of the theorem we need only show that the boundary homomorphism from $\Pi_{2 n-3}\left(S^{n-1}\right)$ to $\Pi_{3 n-p-5}\left(S^{2 n-p-3}\right)$ has a nontrivial kernel for $n \geqq 5, n \neq 8$, and note that, when $n=8$, the boundary homomorphism from $\Pi_{14}\left(S^{7}\right) \cong \mathbf{Z}_{120}$ to $\Pi_{20-p}\left(S^{13-p}\right) \cong \mathbf{Z}_{240}$, if $p>5$, or $\mathbf{Z}+\mathbf{Z}_{120}$, if $p=5$, is a monomorphism whose cokernel is $\mathbf{Z}_{2}$ and $\mathbf{Z}$, respectively, while the boundary homomorphism from $\Pi_{13}\left(S^{7}\right)$ to $\Pi_{19-p}\left(S^{13-p}\right)$ is an isomorphism. If $n$ is even all the elements of $\Pi_{2 n-3}\left(S^{n-1}\right)$ are suspensions and the suspension to $\Pi_{3 n-p-5}\left(S^{2 n-p-3}\right)$ has a nontrivial kernel, $n \geqq 5$, unless $n=8$ when the kernel is trivial. If $n$ is odd $\Pi_{2 n-3}\left(S^{n-1}\right)$ contains an element of infinite order while $\Pi_{3 n-p-5}\left(S^{2 n-p-3}\right)$ is a finite group so that although we do not 
know that the boundary homomorphism is a suspension as above we do know the kernel must be infinite. Thus the theorem is proved.

\section{REFERENCES}

1. D. Burghelea, R. Lashof and M. Rothenberg, Groups of automorphisms of manifolds, Lecture Notes in Mathematics, Springer-Verlag v. 473 (1976).

2. P. J. Hilton, On Introduction to Homotopy Theory, Cambridge University Press, 1964.

3. - On the homotopy groups of the union of spheres, J. London Math. Soc., 30 (1955), 154-172.

4. J. F. P. Hudson, Extending piecewise linear isotopies, Proc. London Math. Soc., (3) 16 (1966), 651-668.

5. K. C. Millett, Piecewise linear concordances and isotopies, Memoir of Amer. Math. Soc. No. 153, 1974.

6. _- Piecewise linear embeddings of manifolds, Illinois J. Math., 19 (1975), 354-369.

7. C. Morlet, Plongements et automorphismes des variétés, Notes Cours Peccot, College de France, 1969.

Received December 23, 1975 and in revised form January 18, 1977. Partially supported by NSF MPS 74-06563.

UNIVERSITY OF CALIFORNIA

SANTA BARbara, CA 93106 


\section{PACIFIC JOURNAL OF MATHEMATICS EDITORS}

ICHARD ARENS (Managing Editor)

niversity of California

os Angeles, CA 90024

\section{. A. BEAUmont}

niversity of Washington

sattle, WA 98105

. C. MOORE

niversity of California

erkeley, CA 94720
J. DUGUNDJI

Department of Mathematics

University of Southern California

Los Angeles, CA 90007

R. FINN AND J. MiLgRAM

Stanford University

Stanford, CA 94305

\section{ASSOCIATE EDITORS}
F. BECKENBACH
B. H. NEUMANN
F. WOLF
K. YosHidA

\section{SUPPORTING INSTITUTIONS}

NIVERSITY OF BRITISH COLUMBIA

UNIVERSITY OF SOUTHERN CALIFORNIA

ALIFORNIA INSTITUTE OF TECHNOLOGY STANFORD UNIVERSITY

NIVERSITY OF CALIFORNIA

ONTANA STATE UNIVERSITY

UNIVERSITY OF HAWAII

NIVERSITY OF NEVADA

UNIVERSITY OF TOKYO

EW MEXICO STATE UNIVERSITY

UNIVERSITY OF UTAH

REGON STATE UNIVERSITY

NIVERSITY OF OREGON

WASHINGTON STATE UNIVERSITY

UNIVERSITY OF WASHINGTON

SAKA UNIVERSITY

AMERICAN MATHEMATICAL SOCIETY

The Supporting Institutions listed above contribute to the cost of publication of this Journal, but they a t owners or publishers and have no responsibility for its contents or policies.

Mathematical papers intended for publication in the Pacific Journal of Mathematics should be in typ Irm or offset-reproduced (not dittoed), double spaced with large margins. Underline Greek letters in re ierman in green, and script in blue. The first paragraph or two must be capable of being used separately as 'nopsis of the entire paper. Items of the bibliography should not be cited there unless absolutely necessary, hich case they must be identified by author and Journal, rather than by item number. Manuscripts, uplicate, may be sent to any one of the four editors. Please classify according to the scheme of Math. Revieu Idex to Vol. 39. All other communications should be addressed to the managing editor, or Elaine Bart Iniversity of California, Los Angeles, California, 90024.

100 reprints are provided free for each article, only if page charges have been substantially pai dditional copies may be obtained at cost in multiples of 50 .

The Pacific Journal of Mathematics is issued monthly as of January 1966. Regular subscription rate: \$72. year (6 Vols., 12 issues). Special rate: $\$ 36.00$ a year to individual members of supporting institutions.

Subscriptions, orders for numbers issued in the last three calendar years, and changes of address should ent to Pacific Journal of Mathematics, 103 Highland Boulevard, Berkeley, California, 94708.

UBLISHED BY PACIFIC JOURNAL OF MATHEMATICS, A NON-PROFIT CORPORATION

Printed at Jerusalem Academic Press, POB 2390, Jerusalem, Israel.

Copyright (C) 1978 Pacific Journal of Mathematics

All Rights Reserved 


\section{Pacific Journal of Mathematics}

\section{Vol. 75, No. $1 \quad$ September, 1978}

Mieczyslaw Altman, General solvability theorems

Denise Amar and Eric Amar, Sur les suites d'interpolation en plusieurs variables ..........................................

Herbert Stanley Bear, Jr. and Gerald Norman Hile, Algebras which satisfy a second order linear partial differential equation ..................

Marilyn Breen, Sets in $R^{d}$ having $(d-2)$-dimensional kernels ............

Gavin Brown and William Moran, Analytic discs in the maximal ideal space

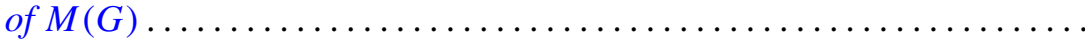

Ronald P. Brown, Quadratic forms with prescribed Stiefel-Whitney

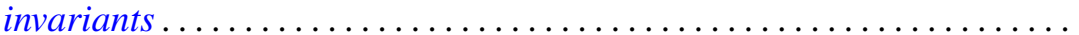

Gulbank D. Chakerian and H. Groemer, On coverings of Euclidean space by convex sets

S. Feigelstock and Z. Schlussel, Principal ideal and Noetherian groups.....

Ralph S. Freese and James Bryant Nation, Projective lattices ............

Harry Gingold, Uniqueness of linear boundary value problems for

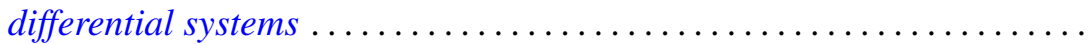

John R. Hedstrom and Evan Green Houston, Jr., Pseudo-valuation domains. . .

William Josephson, Coallocation between lattices with applications to measure extensions

M. Koskela, A characterization of non-negative matrix operators on $l^{p}$ to $l^{q}$ with $\infty>p \geq q>1$

Kurt Kreith and Charles Andrew Swanson, Conjugate points for nonlinear differential equations...........................

Shoji Kyuno, On prime gamma rings ........................ 185

Alois Andreas Lechicki, On bounded and subcontinuous multifunctions ..

Roberto Longo, A simple proof of the existence of modular automorphisms in approximately finite-dimensional von Neumann algebras ...

Kenneth Millett, Obstructions to pseudoisotopy implying isotopy for

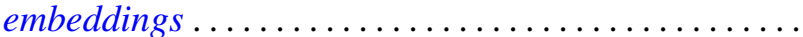

William F. Moss and John Piepenbrink, Positive solutions of elliptic equations. .

Mitsuru Nakai and Leo Sario, Duffin's function and Hadamard's

conjecture

Mohan S. Putcha, Word equations in some geometric semigroups ...

Walter Rudin, Peak-interpolation sets of class $C^{1} \ldots \ldots \ldots$

Elias Saab, On the Radon-Nikodým property in a class of locally convex

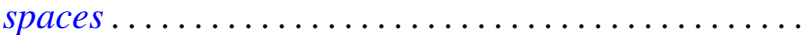

